QUALITY IMPROVEMENT REPORT

\title{
Improving documentation of quality measures in the electronic health record
}

\author{
Peg Esper, DNP, MSN, MSA, ANP-BC, AOCN (Nurse Practitioner) ${ }^{1}$ \& Suzette Walker, DNP, MSN, FNP-C, AOCNP \\ (Nurse Practitioner) $^{2}$ \\ ${ }^{1}$ University of Michigan, Ann Arbor, Michigan \\ ${ }^{2}$ McKenzie Health System, Sandusky, Michigan
}

\author{
Keywords \\ Quality improvement; nurse practitioners; \\ oncology; information technology; palliative \\ care.

\section{Correspondence} \\ Peg Esper, DNP, MSN, ANP-BC, AOCN, \\ University of Michigan, Ann Arbor, MI. \\ E-mail: pegesper@gmail.com \\ Received: 22 April 2013; \\ accepted: 25 August 2013 \\ doi: 10.1002/2327-6924.12169

\section{Disclosures} \\ Preliminary study findings were presented via \\ poster at ONS Connections Conference, \\ Phoenix, AZ-November, 2012 and ASCO \\ Quality Conference, San Diego, CA-December, \\ 2012.
}

\begin{abstract}
Purpose: Oncology quality measures provide an important tool to evaluate care received by cancer patients. These measures are frequently addressed by oncology nurse practitioners (NPs). NP documentation of quality oncology practice initiative (QOPI) measures in the electronic health record (EHR) is evaluated in this study.

Data sources: NP documentation of specific QOPI measures before and after an educational intervention (EI) was evaluated. EHR shortcuts, called "SmartPhrases," were used to increase efficiency in documentation of these measures.

Conclusions: Preintervention chart audits found compliance $<80 \%$ in the multiple measurement areas. Following the EI, NPs surveyed identified greater understanding of QOPI measures and an interest in using "SmartPhrases" to aid in measure documentation. The postintervention audit demonstrated improvement in all areas addressed during the EI noting the use of "SmartPhrases" based on descriptive findings.

Implications for practice: NPs play a significant role in providing quality care for oncology patients. By increasing knowledge related to the documentation of quality measures and providing tools to increase the efficiency associated with their documentation, a positive impact can be made in efforts to promote quality patient care.
\end{abstract}

\section{Purpose}

\section{Introduction}

The provision of quality care is an expectation for oncology practitioners throughout the patient's illness continuum. Improved treatments have significantly lengthened this trajectory and have led to increased survival for many patients with cancer. A new dimension in care, identified as supportive care, focuses on improving the quality of patients' lives during and after treatment. Supportive care includes both palliative care (care that seeks to decrease suffering at all disease stages) and symptom management.

Although a third of patients die within 5 years of a cancer diagnosis, patients with cancer are living longer (American Cancer Society, 2012). This has resulted in patients having more long-term side effects than ever before. In addition, the increase in treatment options and life expectancy has exposed patients to side effects not previously seen in this population. This has re- sulted in an increased demand for quality symptom management for all patients. A landmark randomized trial published by Temel et al. (2010) documented that patients who receive palliative care throughout the course of illness lived longer and reported improved quality of life.

\section{Quality measures in oncology}

In 2002, in an attempt to improve the quality of care for all oncology patients, the American Society of Clinical Oncology (ASCO) developed the quality oncology practice initiative (QOPI). Although supportive care measures were part of the initial measurement set, they were greatly expanded on over the next few years. The QOPI measures were piloted and published by McNiff et al. (2008). These measures represent the only national, systematic, practice-based quality initiative that allows oncology practices to capture data related to supportive care. 
Nurse practitioners (NPs) have a critical role in symptom management and are key providers in assuring incorporation of these quality measures.

\section{Electronic health records and quality measurement}

With the governmental impetus to incorporate evidence of "Meaningful Use" for healthcare system reimbursement, more and more organizations are either moving to or upgrading their electronic health record (EHR). Significant interest lies in the ability to use the EHR to capture documentation of a variety of measures, including quality measures. To date, several studies have evaluated this with mixed responses. In a study involving primary care clinics by Linder, Kaleba, and Kmetik (2009), EHR encounters for 688 patients with a claim diagnosis of pneumonia, were reviewed. Wide variation in performance measurement was noted and accurate identification of quality measures in the EHR was noted to be challenging. Another interesting finding was noted in a retrospective study by Parsons, McCullough, Wang, and Shih (2012), in which over 4000 records across 57 practices were reviewed to determine the validity of EHR-derived quality measures following a comprehensive training program. Their findings showed that the EHR-derived reporting could have a disproportionately negative impact on the ability to capture this information based on workflows and other payor-based requirements for documentation.

Persell et al. (2011), however, did find in a large time series that EHR tools could be used to accelerate improvement in performance of quality measures based on a quality improvement intervention that included clinician feedback. The quality improvement study addressed inefficiencies in the EHR and included a mechanism to inform clinicians when quality measures were not being met (such as important medications not being received by patients). Overall, there remains a paucity in the literature related to the relationship between staff education and improving documentation of quality measures via an EHR.

The purpose of this quality improvement study was to enhance the current knowledge level of oncology NPs within an academic NCI-Designated Comprehensive Cancer Center related to quality measures in symptom management and end-of-life care; and support documentation of measures in an EHR by incorporating "SmartPhrases" (a documentation shortcut specific for the university's EHR software program that allows the typing in of a "cue" phrase that will populate a larger body of documentation) that capture quality measures content. Improved implementation and documentation of care processes pave the way for future measurement of patient outcomes.
This project utilized quality measures that are included in QOPI chart reviews. Neuss et al. (2005) reported that by using the QOPI process, a rapid and objective measurement of practice quality is obtained. QOPI has been shown to provide a tool to practice self-examination that can promote excellence in cancer care.

\section{Data sources}

Approval for this research was obtained from the university's Institutional Review Board. Permission was also obtained from ASCO to utilize QOPI measures to gather data. The QOPI abstraction tool was modified to include specific measures from modules that addressed pain, emotional well-being, end-of-life care, and emetogenic chemotherapy.

The database for the cancer center was queried for the records of patients seen by Cancer Center Medical Oncology NPs during the period of January to March 2012. Data were not used from surgical oncology, radiation oncology, or bone marrow transplant. A random numbers chart was used to assign NP charts between the two nurse researchers who performed the independent chart audits. Then, a random number chart was used to choose five charts of patients seen by each NP for the preintervention audit. Several test charts were reviewed by both researchers to evaluate for interrater reliability that was $100 \%$.

One hundred medical charts of Cancer Center patients were retrospectively reviewed for documentation of select supportive care QOPI measures. This was completed in August of 2012. Data were entered and analyzed using SPSS software. Areas of deficiency in documentation were identified and used to develop an educational intervention (EI) for the NP staff. The areas to be used in the EI were based on a selected $80 \%$ compliance level.

Based on this assessment, an intensive EI was developed. This incorporated a didactic presentation and interactive case studies and was subsequently presented to NPs within the Cancer Center. "SmartPhrases" were developed to support the documentation of the QOPI measures found to be below the established compliance level. Reminder cards were developed listing the "SmartPhrases" and given to all NPs during the educational sessions (Figure 1). These "SmartPhrases" were developed by the investigators by using the institution's EHR personalization tools (Epic, Verona, WI). To encourage attendance to the presentations, multiple offerings were scheduled for the presentations, food was offered at all sessions, and a gift card was raffled off for attendees. A survey was distributed to the NPs at the end of each session to evaluate their level of knowledge regarding QOPI measures 


\section{QOPI SmartPhrases To Remember}

.QOPIPAINEVAL- Document eval/action for scores $\geq 4$

.QOPIPAINRV - Document follow up after Rx for Pain

.QOPIBOWELEVAL - Document initial bowel assessment

.QOPIEMOEVAL - Document initial emotional assessment

.QOPIEMOHELP - Document action $r / t$ emotional status

Figure 1 "SmartPhrase" reminder cards.

and their likelihood to utilize the "SmartPhrases" provided to document these measures. Reminder e-mails were sent out weekly to the NPs who attended the EI to promote incorporation of measures into documentation.

Four weeks following the EI, another chart audit was performed to assess the intervention's effectiveness. Only charts of those NPs that attended the educational session were audited. Five charts per NP were audited for a total of 65 charts being assessed using the same strict criteria as prior. Analysis of post-EI data commenced in November, 2012.

\section{Implications for practice}

Descriptive statistics were used to evaluate data obtained during the pre- and post-EI chart audits. SPSS and Excel software programs were utilized for data management.

\section{Pre-El chart audit}

The "pre" EI chart audit of 100 records was reviewed for measures that fell below an $80 \%$ compliance level. The authors adhered to a stringent and somewhat expanded definition of each measure in an effort to identify where intervention would be most meaningful. Those measures falling below the established threshold included

- Documentation of the plan for addressing moderate to severe pain.

- Appropriateness of the management plan for moderate to severe pain.

- Assessment of narcotic efficacy on the return visit following initial or prescription change.

- Assessment of bowel function at the time of narcotic prescription.

- Assessment of bowel function postnarcotic prescription.

- Assessment of emotional well-being.

- Plan for addressing emotional well-being, if indicated.
Measures addressing oral chemotherapy management also fell below the $80 \%$ level. A new oral chemotherapy program was initiated following the preintervention chart audit at the study institution. As a result, these measures were not selected for inclusion in the EI as the investigators felt this would introduce too many confounding variables. Indicators regarding the appropriate supportive measures for patients receiving moderate or highly emetogenic chemotherapy exceeded the threshold for inclusion in the EI. Results of the pre-EI chart audits are detailed in Figure 2.

\section{EI}

Overall, a total of 18 advanced practice nurses attended one of the EIs ( 13 medical oncology NPs, two surgical oncology NPs, one psych oncology NP, one clinical nurse specialist, and one NP supervisor). A brief survey was administered to attendees following the session to evaluate their initial impression of the information provided. Questions and responses were as follows:

- Following this educational session, I have a better understanding of what QOPI is: Yes-94\%, Somewhat-6\%

- Following this educational session, I have an understanding of how using "SmartPhrases" can improve my documentation: Yes-100\%

- Following this educational session, I will use "SmartPhrases" to improve my documentation: Yes-72\%, Maybe-28\%

- I believe that better documentation will improve patient care: Yes-78\%, Somewhat-22\%

The investigators found the NP staff who attended the sessions to be interested in learning more about how quality measures could be incorporated into their documentation. In general, they had minimal knowledge regarding QOPI measures and did not realize that patient charts were routinely being abstracted to evaluate documentation of these measures. Several NP staff attending sessions asked for results of their personal chart audit to be shared with them.

\section{Post-El chart audit}

Results of the post-EI chart audit are seen and compared with preintervention chart audit findings in Figure 2. Each of the nine indicators addressed during the EI showed an improved level of compliance at the time of the postintervention chart audits. While not quantitatively measured, investigators observed that a number of NPs appeared to utilize the "SmartPhrases" from the reminder cards provided to them as part of the 


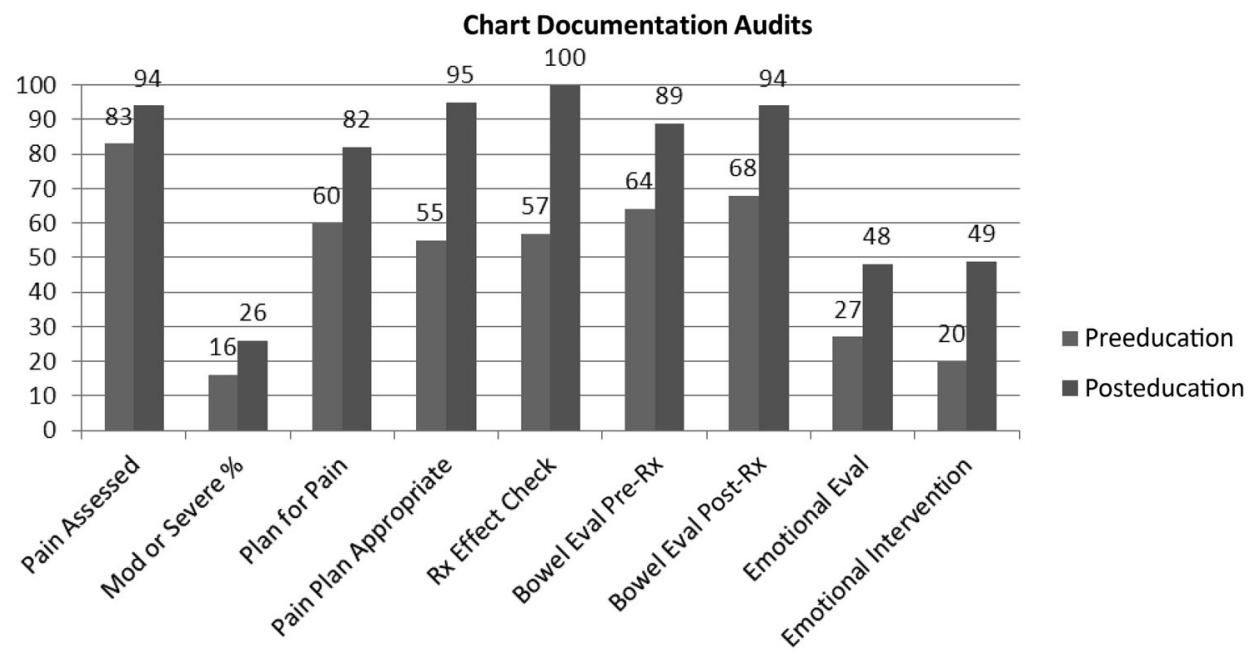

Figure 2 Pre- and post-El chart audit results.

Note. Results expressed in percentage of charts with completed documentation (pre-El, $n=100$; post-El, $n=65$ ).

educational sessions. Once the "SmartPhrase" was placed in a progress note, it can be modified. As a result, the authors' ability to quantify that the exact use of "SmartPhrases" was not possible.

The greatest degree of improvement was noted in documentation of measures to intervene for identified emotional concerns ( $145 \%$ increase). In addition, a greater than $70 \%$ improvement was seen in the documentation of an appropriate plan for pain management, the effectiveness of the pain management intervention at the subsequent visit, and evaluation of patient emotional status.

The final results of this project were shared at a forum for advance practice nurses throughout the authors' workplace. While this included "non-oncology" APNs, the intent was to demonstrate how documentation of quality measures can be impacted when an EHR is in use and strategies that can be used to facilitate documentation. The project was well received and future discussion may take place with the EHR vendor to try and incorporate some of these SmartPhrases into the current system more efficiently.

\section{Limitations}

While the investigators in this study were very encouraged by the post-EI chart audit, several limitations to this study are acknowledged. The sample utilized for the study was small. It included only one academic institution and focused on one provider segment-medical oncology NPs.
This alone makes the findings from the study difficult to generalize to other institutions. It also increased the difficulty of finding appropriate charts for the chart audits. In a large teaching facility, the NP may not always be the provider to see the patient at subsequent visits. In addition, the investigators chose the QOPI measures believed to be impacted most by NPs in the initial chart audit. This may have also introduced bias and difficulty in the generalization of findings. Most importantly, the investigators are NPs in the institution and well known to the participants in this study. Attendance at the educational session was likely influenced by this. It is also plausible that the improvement seen in the postchart audit was based on the fact that the NPs in attendance were aware of the fact that charts would be reaudited following the educational session. This may have influenced their documentation of measures addressed during the educational sessions via a Hawthorn effect.

Another important limitation of this study was the introduction of the new electronic medical record shortly prior to the EI. Staff was admittedly stressed as a result of the change in documentation. Asking them to participate in this quality improvement process at the time it was offered may have caused additional stress and affected outcomes. The post-EI chart audit was completed in a time frame relatively close to the intervention and an audit done 46 months following the intervention may have resulted in different findings, and also demonstrated whether persistence in quality measure documentation had occurred. 


\section{Conclusions}

This project included a number of important goals. These included determining the degree to which quality measures for symptom management and end-of-life care are incorporated into NP practice, increasing NP knowledge related to established oncology quality measures for symptom management and end-of-life care, and evaluating change in the use of established quality measures for symptom management and end-of-life care following a designed EI. While many administrative staff may believe that nationally vetted standards are automatically part of the staff's knowledge base, this is often not the case. Quality measures, such as the QOPI measures, are not necessarily discussed on a regular basis and institutional reports may not be shared at the staff level. This study introduced QOPI measures to the NP staff and increased their awareness of how the measures were developed, the role of the provider in documenting these measures, with an emphasis on the importance of the adage, "if it wasn't documented, it wasn't done." Case studies using real patient encounters allowed staff to see how the quality measures could be readily incorporated into documentation and allow for improved continuity of care in future visits. Sharing the deficits seen during the prechart audit with the NP staff provided an impetus for them to think about their own style and depth of documentation.

The time providers have for documentation is constantly being impacted by the many other areas to be addressed during a very time-limited patient encounter. Providing staff with tools that can help expedite documentation has the potential to improve the quality of information in the medical record. Administrators would be well served to evaluate the standards and quality measures that are pertinent to their organization as new EHRs are being implemented and to strive to incorporate these into user templates. The attempt of this study to aid documentation of quality measures by the use of "SmartPhrases" did prove to be something that staff was able to incorporate into their practice. By making these phrases easily accessible and available to all staff, it increases the likelihood that improvement in the documentation of the quality measures will be seen.

Providing quality oncology care to patients is a major goal of care. A number of national guidelines, such as the QOPI measures, have been created in an attempt to establish standards for consistently providing quality patient care. Efforts should continue to evaluate the most optimal ways for these measures to be implemented and documented. Quality improvement projects, such as the one described in this paper, represent an important step in the process of improving patient outcomes.

\section{Acknowledgments}

The authors thank Constance Creech, RN, EdD, ANP-BC, Associate Professor of Nursing, University of Michigan-Flint, and Mary B. Killeen, RN, PhD, CNAA-BC, Adjunct Associate Professor of Nursing, University of Michigan-Flint. This study was completed as partial fulfillment of the DNP degree for the authors at the University of Michigan-Flint. Drs. Creech and Killeen served on the Capstone Committee for the authors.

\section{References}

American Cancer Society. (2012). Cancer facts $\theta$ figures 2012. Atlanta, GA: American Cancer Society.

Linder, J. A., Kaleba, E. O., \& Kmetik, K. S. (2009). Using electronic health records to measure physician performance for acute conditions in primary care: Empirical evaluation of the community-acquired pneumonia clinical quality measure set. Medical Care, 47(2), 208-216. doi:10.1097/MLR.0b013e318189375f

McNiff, K. K., Neuss, M. N., Jacobson, J. O., Eisenberg, P. D., Kadlubek, P., \& Simone, J. V. (2008). Measuring supportive care in medical oncology practice: Lessons learned from the quality oncology practice initiative. Journal of Clinical Oncology: Official Journal of the American Society of Clinical Oncology, 26(23), 3832-3837. doi:10.1200/JCO.2008.16.8674

Neuss, M. N., Desch, C. E., McNiff, K. K., Eisenberg, P. D., Gesme, D. H., Jacobson, J. O., ... Simone, J. V. (2005). A process for measuring the quality of cancer care: The quality oncology practice initiative. Journal of Clinical Oncology: Official Journal of the American Society of Clinical Oncology, 23(25), 6233-6239. doi:10.1200/JCO.2005.05.948

Parsons, A., McCullough, C., Wang, J., \& Shih, S. (2012). Validity of electronic health record-derived quality measurement for performance monitoring. Journal of the American Medical Informatics Association: JAMIA, 19(4), 604-609. doi:10.1136/amiajnl-2011-000557

Persell, S. D., Kaiser, D., Dolan, N. C., Andrews, B., Levi, S., Khandekar, J., ... Baker, D. W. (2011). Changes in performance after implementation of a multifaceted electronic-health-record-based quality improvement system. Medical Care, 49(2), 117-125. doi:10.1097/MLR.0b013e318202913d

Temel, J. S., Greer, J. A., Muzikansky, A., Gallagher, E. R., Admane, S., Jackson, V. A., ... Lynch, T. J. (2010). Early palliative care for patients with metastatic non-small-cell lung cancer. New England Journal of Medicine, 363(8), 733-742. doi:10.1056/NEJMoa 1000678 